\title{
3D multicellular models reflect the efficiency of MSC-directed enzyme/ prodrug treatment
}

\author{
R. BOHOVIC, L. DEMKOVA, M. CIHOVA, S. SKOLEKOVA, E. DURINIKOVA, L. TORO, S. TYCIAKOVA, Z. KOZOVSKA, M. MATUSKOVA, \\ L. KUCEROVA*
}

Laboratory of Molecular Oncology, Cancer Research Institute, Slovak Academy of Sciences, Bratislava, Slovakia

${ }^{*}$ Correspondence: lucia.kucerova@savba.sk

Received November 24, 2014 / Accepted December 15, 2014

\begin{abstract}
Mesenchymal stromal cells (MSC) exhibit beneficial properties to serve as cellular vehicles for enzyme/prodrug cancer gene therapy approaches. We have previously shown that engineered human adipose tissue-derived MSC in combination with non-toxic prodrug mediated substantial cytotoxic and antitumor effect. The aim of this study was to develop advanced $3 \mathrm{D}$ cultivation method to serve for modelling of the therapeutic outcome in vitro. We have used engineered MSC expressing fusion transgene cytosine deaminase::uracilphosphoribosyltransferase (CD-MSC) in combination with prodrug 5-fluorocytosine (5FC). This therapeutic regimen designated CD-MSC/5FC was combined with the human melanoma cells A375 or EGFP-A375 in both standard monolayer culture and 3-dimensional (3D) multicellular nodules. The extent of cytotoxicity was evaluated by standard viability assay MTS, ATP-based luminescence assay, fluorimetric test, measurement of Caspase-3/7 activation and DNA laddering. The data have shown that the extent of cytotoxic bystander effect mediated by CD-MSC/5FC is significantly lower in 3D culture conditions. However, these data better recapitulate the therapeutic efficiency as observed previously in vivo. We suggest here to use the 3D multicellular culture conditions for better prediction of the therapeutic outcome in mouse xenograft models.
\end{abstract}

Key words: human mesenchymal stromal cells, adipose tissue, melanoma, multicellular nodule, 3D culture

Mesenchymal stromal cells (MSC) are found within all mammalian supportive stromal tissue compartments in situ. They can be isolated by their adherence to plastic and consecutive passaging. MSC proliferate in vitro to form heterogeneous population of fibroblast-like cells that are at varying stages of commitment to differentiation [1]. The minimal criteria define these cells by a combination of surface markers and trilineage differentiation potential. Bone marrow, adipose tissue and the umbilical cord are the most commonly used as the cell source for the MSC isolation. The MSC possess an immune-privileged status, and preferential homing ability for injured, inflamed or tumor tissue [2]. Beneficial inherent MSC properties such as their availability, genotypic and phenotypic stability, expandability and intratumoral distribution can be exploited for the antitumor treatment. Therefore, several reports including our own data suggested that the MSC might serve as suitable delivery vehicles for the proteins of interest into tumors [3;4]. The exogenous MSC are capable of selective homing to and surviving in a variety of pre-established solid tumors (breast, colon, melanoma etc.) or metastases [5; 6]. Moreover, MSC were not detectable in a healthy mouse tissue(s) after 7 days post systemic administration [7].

In the light of the experimental evidence showing the contribution of the MSC to tumor stroma and progression [8], it seems the MSC are optimal cells for the gene-directed enzyme/prodrug therapy approaches. The gene of interest expressed in MSC such as cytosine deaminase or herpes simplex virus -thymidine kinase (HSV-TK) in combination with respective non-toxic prodrug (5-fluorocytosine, 5FC, or ganciclovir, GCV, respectively) mediates production of toxic antitumor metabolites at the site of tumor growth and exerts bystander cytotoxicity eliminating tumor cells. The same gene concomitantly causes a suicide effect thereby eliminating the engineered MSC from the site of the tumor growth soon after the prodrug administration. Thus the cytotoxicity and antitumor action is targeted to the site of the tumor growth and temporally limited by the inherent suicide gene function $[9 ; 10]$. However, these approaches have their 
limitations and we have identified several biomarkers in the tumor cells which contribute to the efficiency of the treatment regimen [11].

Based on our experimental evidence we have shown that melanoma cells A375 exhibit superior sensitivity to CDMSC/5FC treatment regimen in vitro [6]. These melanoma cells express high levels of thymidylate synthase and thymidine phosphorylase (enzymes of the nucleotide mechanism) which significantly contribute to the incorporation of the 5FC-metabolites into DNA and RNA thus efficiently mediating the cytotoxicity [11]. Our data also indicated that low expression levels of multidrug resistance gene(s) MRP5 (ABCC5) and MRP8 (ABCC11) might contribute to A375 cell sensitivity to the CD-MSC/5FC combination. Standard MTS viability assay revealed that the $\mathrm{IC}_{50}$ value for the $\mathrm{A} 375$ cells was $0.01 \%$; in other words, it was sufficient to admix one therapeutic CD-MSC cell to 10,000 target melanoma cells to achieve $50 \%$ decrease in viability in the presence of $5 \mathrm{FC}$ within 5 days [6]. Furthermore, the therapeutic-to-target cell ratio 1:20 CD-MSC/5FC:A375 decreased in vitro by $90 \%$ viability as determined by the standard MTS viability assay. These data correlated with the outcomes from the fluorimetric evaluation of the bystander effect and luminometric viability assay based on the ATP levels in the treated cells [12]. In contrast, it was necessary to co-inject $10 \%$ of therapeutic CD-MSC cells with the target melanoma A375 cells in order to achieve significant delay in the tumor onset. Twenty per cent of therapeutic cells in coinjected mixtures were necessary to achieve a long-term tumor-free survival and a complete tumor eradication in $83.3 \%$ of the xenografted immunocompromised mice. Thus it is obvious that the therapeutic efficiency of the given treatment is lower in vivo in comparison to the outcomes as suggested by coculture experiments in vitro.

In the present study our efforts were focused on a 3-dimensional coculture system in multicellular nodules as a potential model for the evaluation of therapeutic outcome. Based on our knowledge this is a first study to examine bystander effect mediated by cytosine deaminase and $5 \mathrm{FC}$ in the $3 \mathrm{D}$ coculture models. Indeed, our data confirm that the 3D coculture systems can be efficiently used for prediction of the therapeutic activity of the cell-based therapy in vivo.

\section{Material and methods}

Cells and chemicals. All chemicals were purchased from Sigma (St. Louis, MO) if not stated otherwise.

Human melanoma cells A375 (ECACC No. 88113005) and EGFP-A375 were cultured in high-glucose $(4.5 \mathrm{~g} / \mathrm{L})$ Dulbecco's modified Eagle medium (DMEM, PAA Laboratories Inc., Etobicoke, ON, Canada) supplemented with $5 \%$ fetal bovine serum (FBS, Biochrom GmbH, Berlin, Germany), $2 \mathrm{mM}$ glutamine and Antibiotic-antimycotic $\left(\right.$ GIBCO $^{\circ}$ Invitrogen $^{\text {Twx }}$ Life Technologies Carlsbad, CA). A375 cell line stably expressing enhanced green fluorescent protein (EGFP) was prepared as described elsewhere [13].
EGFP expression was confirmed by fluorescent microscopy and flow cytometry on the BD Canto II cytometer (Becton Dickinson, Franklin Lakes, NJ, USA) equipped with the FACSDiva program. FCS Express software was used for the evaluation.

Human adipose tissue-derived mesenchymal stromal cells were isolated by collagenase digestion from lipoaspirate obtained from healthy persons, who provided an informed consent as established previously [5]. Therapeutic CD-MSC were prepared, expanded and characterized as previously described in detail $[5 ; 6]$. CD-MSC were expanded in lowglucose DMEM (1 g/L) with 5\% GlutamMAX ${ }^{\text {tw }}$ (GIBCO Invitrogen Life Technologies) supplemented with $10 \% \mathrm{Hy}$ Clone $^{\infty}$ AdvanceSTEM $^{\mathrm{m}}$ Mesenchymal Stem Cells Growth Supplement (Thermo Scientific, Waltham, MA) and Antibiotic-antimycotic (GIBCO ${ }^{\circ}$ Invitrogen ${ }^{\text {mo }}$ Life Technologies). Cells were maintained at $37^{\circ} \mathrm{C}$ in $5 \% \mathrm{CO}_{2}$ humidified atmosphere. MSC were characterized by tri-lineage differentiation as described previously [14] and surface marker expression as CD73+, CD90+, CD105+, CD14-, CD20-, CD34-, CD45- population by MSC phenotyping kit (Miltenyi Biotec, Bergisch Gladbach, Germany).

3D cultures. Multicellular nodules were prepared as previously described [15]. Briefly, monolayer cultures of CD-MSC and tumor cells were dissociated and mixed at a desired ratio to obtain $5 \times 10^{5} \mathrm{cells}$. Cells were then centrifuged at 1,200 rpm for $10 \mathrm{~min}$ in sterile centrifuge tubes containing $0.25 \mathrm{ml}$ of semi-solid culture medium ( $0.5 \%$ agar in culture medium). Cell pellets were incubated overnight at $37^{\circ} \mathrm{C}, 5 \% \mathrm{CO}_{2}$. The next day, cell pellets were supplemented with or without 5FC-containing medium. Nodules were let to grow until used for further analysis. Conclusions were drawn from two independent experiments performed and evaluated in at least triplicates.

Alternatively, quadruplicates of $1 \times 10^{4}$ EGFP-A375 were mixed with CD-MSC as indicated and seeded into 96-well ultra-low attachment plates (Corning 7007, Corning Inc., NY, USA) in $100 \mu$ of standard culture medium. Forty eight hours later, $100 \mu \mathrm{l}$ of media with or without 5FC at indicated concentration was added to the wells and the spheroid growth was monitored every 6 hours by IncuCyte $\mathrm{ZOOM}^{\mathrm{Tm}}$ Kinetic Imaging System (Essen BioScience, Welwyn Garden City, UK). Data were evaluated according to the Cell Player $^{\mathrm{m}}$ 96well kinetic 3D spheroid protocol as recommended by the manufacturer. At the assay endpoint, plates were subjected to the viability assays as described below.

Transgene expression in CD-MSC. Total RNA was isolated from $2 \times 10^{6}$ cells by RNeasy mini kit (Qiagen, Hilden, Germany) and treated with RNase-free DNase (Qiagen, Hilden, Germany). Five $\mu \mathrm{g}$ RNA was reverse transcribed with RevertAid ${ }^{\mathrm{TM}}$ H Minus First Strand cDNA Synthesis Kit (Fermentas, Hanover, MD).

PCR was performed in 1x PCR Master Mix (Fermentas, Hanover, MD), $2.5 \mathrm{mM} \mathrm{MgCl}, 0.16 \mu \mathrm{M}$ primers, and template cDNA (1-2 $\mu \mathrm{g})$. Following primers were used: FcyFOR 
5'-Atggacattgcctatgagga-3' and FcyREV 5'- TTCTCCAGGGTGCTGATCTC-3' for cytosine deaminase (167 bp amplicon), GAPDHF or 5'-GAAGGTGAAGGTCGGAGTC-3' and GAPDHRev 5'-GAAGATGGTGATGGGATTTC-3' for GAPDH to (226 bp amplicon). Template cDNA was subjected to the standard PCR with 35 cycles and the samples were resolved on $2 \%$ agarose gel. To detect CD transgene in transduced MSC, the genomic DNA isolated from MSC and CD-MSC (500 ng) was subjected to PCR with primers FcyFOR and FcyREV and analysed as above.

5-Fluorocytosine conversion. The cytosine deaminase enzyme activity was measured spectrophotometrically [16]. Two $x 10^{6}$ cells were resuspended in $100 \mu$ of PBS, lysed by five cycles of rapid freeze-and-thaw in liquid nitrogen. Lysates were centrifuged at $15,000 \mathrm{rpm}$ for $30 \mathrm{~min}$ at $4^{\circ} \mathrm{C}$. Fifty $\mu \mathrm{l}$ of clear supernatant was mixed with $50 \mu \mathrm{lof} 0.4 \mathrm{mg} / \mathrm{ml} 5 \mathrm{FC}$ in PBS and incubated at $37^{\circ} \mathrm{C} .10 \mu \mathrm{l}$ aliquots were taken at each timepoint, quenched with $90 \mu \mathrm{l}$ of $0.1 \mathrm{M} \mathrm{HCl}$, and spectrophotometrically evaluated on Nanodrop-1000 (Wilmington, DE). Enzymatic conversion of 5FC was calculated as per cent of 5FC remaining in the lysate based on the following equation:

$5 \mathrm{FC}$ remaining $(\%)=\left[0.119 * \mathrm{OD}_{290}(0)-0\right.$ $\left..025^{\star} \mathrm{OD}_{255}(0)\right]-\left[0.119^{\star} \mathrm{OD}_{290}(\mathrm{x})-0.025^{\star} \mathrm{OD}_{255}(\mathrm{x})\right] /$ $\left[0.119^{\star} \mathrm{OD}_{290}(0)-0.025^{\star} \mathrm{OD}_{255}(0)\right]$, where $\mathrm{OD}_{290}(0)$ and $\mathrm{OD}_{255}(0)$ are starting absorbance values (timepoint 0 ) at 290 $\mathrm{nm}$ and $255 \mathrm{~nm}$ respectively, and $\mathrm{OD}_{290}(\mathrm{x})$ and $\mathrm{OD}_{255}(\mathrm{x})$ are absorbance values at $\mathrm{x}$ hrs at $290 \mathrm{~nm}$ and $255 \mathrm{~nm}$, respectively. Conversion of 5FC was expressed as means of triplicates \pm SD and indicates per centage of starting $5 \mathrm{FC}$ concentration that was set to $100 \%$ by default.

DNA laddering. The method was adopted from Zhang et al. [17]. The cells from monolayers or nodules $\left(2 \times 10^{6}\right)$ were collected and centrifuged at $1,400 \mathrm{rpm}$ for $10 \mathrm{~min}$ at room temperature. Then they were washed with PBS and lysed in 1 $\mathrm{ml}$ of lysis buffer (Tris- $\mathrm{HCl} 10 \mathrm{mM}, \mathrm{pH}=7.4$, EDTA $10 \mathrm{mM}$, $0.5 \%$ Triton $\mathrm{X}-100$ and proteinase $\mathrm{K} 40 \mu \mathrm{g} / \mathrm{L}$ ) at $37^{\circ} \mathrm{C}$ for 2 hrs. The lysate was extracted with $0.5 \%$ solution of $5 \mathrm{M} \mathrm{NaCl}$ and $50 \%$ isopropanol. DNA was precipitated overnight at $-20^{\circ} \mathrm{C}$ and centrifuged at $10,000 \mathrm{rpm}$ for $20 \mathrm{~min}$. Pellet was washed with $70 \%$ ethanol, dried and dissolved in TE buffer (Tris- $\mathrm{HCl} 10 \mathrm{mM}, \mathrm{pH}=7.4$, EDTA $1 \mathrm{mM}$ ). DNA was treated with RNase A ( $40 \mu \mathrm{g} / \mathrm{L})$ at $37^{\circ} \mathrm{C}$ for $60 \mathrm{~min}$. DNA was resolved on $2 \%$ agarose gel.

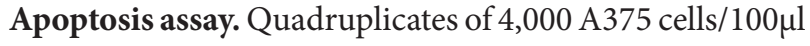
media/well were seeded in 96-well white-walled plates $24 \mathrm{hrs}$ prior to treatment start. Nodule A375 cultures were initiated as described above. Both monolayer and nodule cultures were treated with $5 \mathrm{FU}$ at given concentrations for $24 \mathrm{hrs}$. Caspase3/7 activity was determined by the ApoTox-Glo ${ }^{\mathrm{mm}}$ Assay (Promega Corporation, Madison, WI). Nodule structures were lysed in the reagent solution and aliquots were subsequently transferred to 96-well white-walled plates for measurement on a LUMIstar GALAXY reader (BMG Labtechnologies, Offenburg, Germany). Values were expressed as an average of relative luminescence units (RLU) $\pm S D$.
Cell viability assays. MTS assay. Quadruplicates of 4,000 cells/200 $\mu \mathrm{l}$ media/well were seeded in 96-well plates for each treatment. Treatments were initiated 24 hours after plating with appropriate drug dilutions in fresh medium. Medium was discarded at the day of evaluation and relative viability of cells was assessed by the CellTiter ${ }^{\circledR} 96$ Aqueous One Solution Cell Proliferation Assay (MTS Assay, Promega). $10 \mu \mathrm{l}$ of 5 $\mathrm{mg} / \mathrm{ml}$ MTS diluted in $90 \mu \mathrm{l}$ DMEM was added to each well of 96-well plate and incubated for $2 \mathrm{hrs}$ at $37^{\circ} \mathrm{C}$. Absorbance values were read out at $492 \mathrm{~nm}$ on a plate reader X-MARK (Bio-Rad, Hercules, CA, USA). The experiments were performed twice in triplicates, and the representative outcome is shown. Results were expressed as relative viability, where the OD corresponding to the viability of untreated tumor cells in standard culture medium DMEM without CD-MSC was set to $100 \%$ by default.

Fluorimetric assay. Quadruplicates of 3,500 EGFP-A375 cells/100 $\mu \mathrm{lmedia} /$ well mixed with $\mathrm{CD}-\mathrm{MSC}$ at given proportion were seeded in black-walled 96-well plates (Greiner Bio-One Intl. AG, Kremsmunster, Austria) overnight. For the 5FC treatments, $100 \mu \mathrm{l}$ culture medium with the 5FC diluted to the final concentration of $100 \mu \mathrm{g} / \mathrm{ml}$ was added to the cells for indicated treatment time. At the experiment endpoint, culture wells were washed with PBS and cells were permeabilized with $0.2 \%$ Nonidet P-40 in PBS. Relative fluorescence was evaluated by the PolarStar OPTIMA reader (BMG Labtechnologies, Offenburg, Germany). Values were expressed as means of relative fluorescence \pm SD, where the fluorescence of untreated EGFP+ cells was set to $100 \%$ by default.

Luminometric assay. Alternatively, the relative viability of the cells in the experimental setup as above was examined by the CellTiter-Glo ${ }^{\text {Tix }}$ Luminescent Cell Viability Assay (Promega Corporation, Madison, WI) and evaluated by the LumiStar GALAXY reader (BMG Labtechnologies, Offenburg, Germany) according to the recommendation of manufacturer. Experiments were performed at least four times with similar results and the representative result is shown, values are expressed as means $\pm \mathrm{SD}$.

Assays in $3 D$ nodules. Nodules were carefully washed with PBS, lysed with $0.2 \%$ Nonidet P- 40 in PBS and the supernatant was transferred to black-walled 96-well plates for the fluorimetric test. Nodules from independent experiments were incubated with luminescent reagent solution from the Luminescent Cell Viability Assay and aliquots were transferred to white-walled 96well plates. One hundred $\mu \mathrm{l}$ of media from 3D spheroids grown in ultra-low attachment plates was discarded, $100 \mu$ of lysis or luminescent reagent solution was added to respective wells and supernatants were transferred to the black or white-walled plate for the measurement in 96-well format as described above.

Statistical analysis. The Student's two-sample t-test was used for hypothesis testing for the difference in means of two samples, assuming that both samples come from a normal distribution with the standard deviations unknown but assumed equal. The $\mathrm{p}$-values with $\mathrm{p}<0.05$ were considered to be statistically significant. 
Results

We have used human adipose tissue-derived mesenchymal stromal cells (MSC) throughout the study. These cells were derived from healthy young donors who underwent elective surgery. As described in several reports from our group, MSC were derived by collagenase digestion method and subsequent expansion of plastic adherent cells. MSC were routinely characterized for the typical surface markers after two rounds of passaging and also subjected to the standard differentiation protocol to confirm the tri-lineage differentiation in vitro (data not shown). Retroviral transduction and subsequent selection of stable-transfectants resulted in generation of genetically engineered CD-MSC, which was confirmed by the presence of integrated CD transgene in the genome (Fig. 1A). These cells constitutively expressed high levels of the transgene (Fig. 1B) and they were able to metabolize prodrug 5-fluorocytosine (5FC) as determined by the spectrophotometric method (Fig. 1C). Enzymatic conversion of 5FC generated phosphorylated derivatives of 5-fluorouracil (5FU) exerted suicide cytotoxic effect on the therapeutic CD-MSC cells (Fig. 1D). MTS assay confirmed that 5FC was not toxic for the naïve MSC; however, $\mathrm{IC}_{50}(5 \mathrm{FC})=1 \mu \mathrm{g} / \mathrm{ml}$ in CD-MSC after 5 days of incubation.

Previously we have reported superior efficiency of the therapeutic paradigm CD-MSC/5FC on melanoma cells A375 in direct monolayer cocultures in vitro $[6 ; 12]$. Based on the data published by Benouchan et al. [15], we adopted their protocol to examine the therapeutic efficiency on the multicellular nodules as a model for early non-vascularized tumors in vitro.

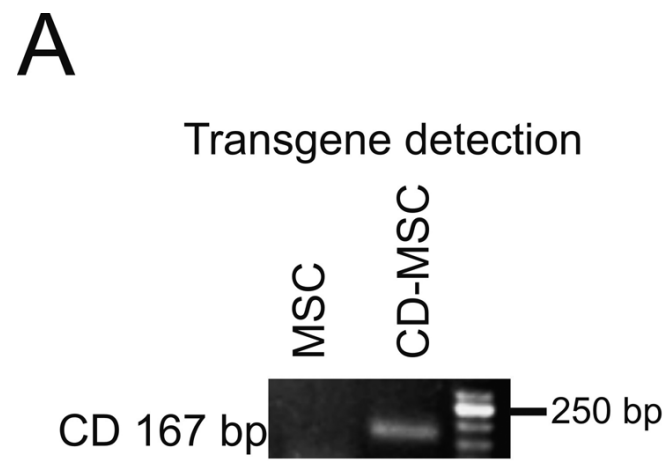

Transgene expression

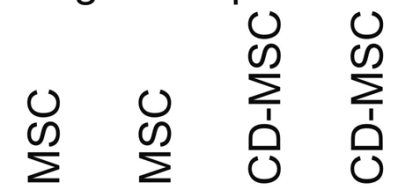

CD

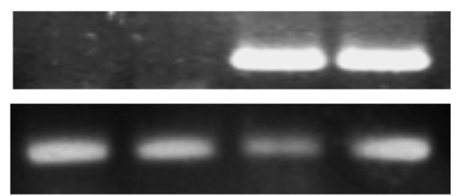

C
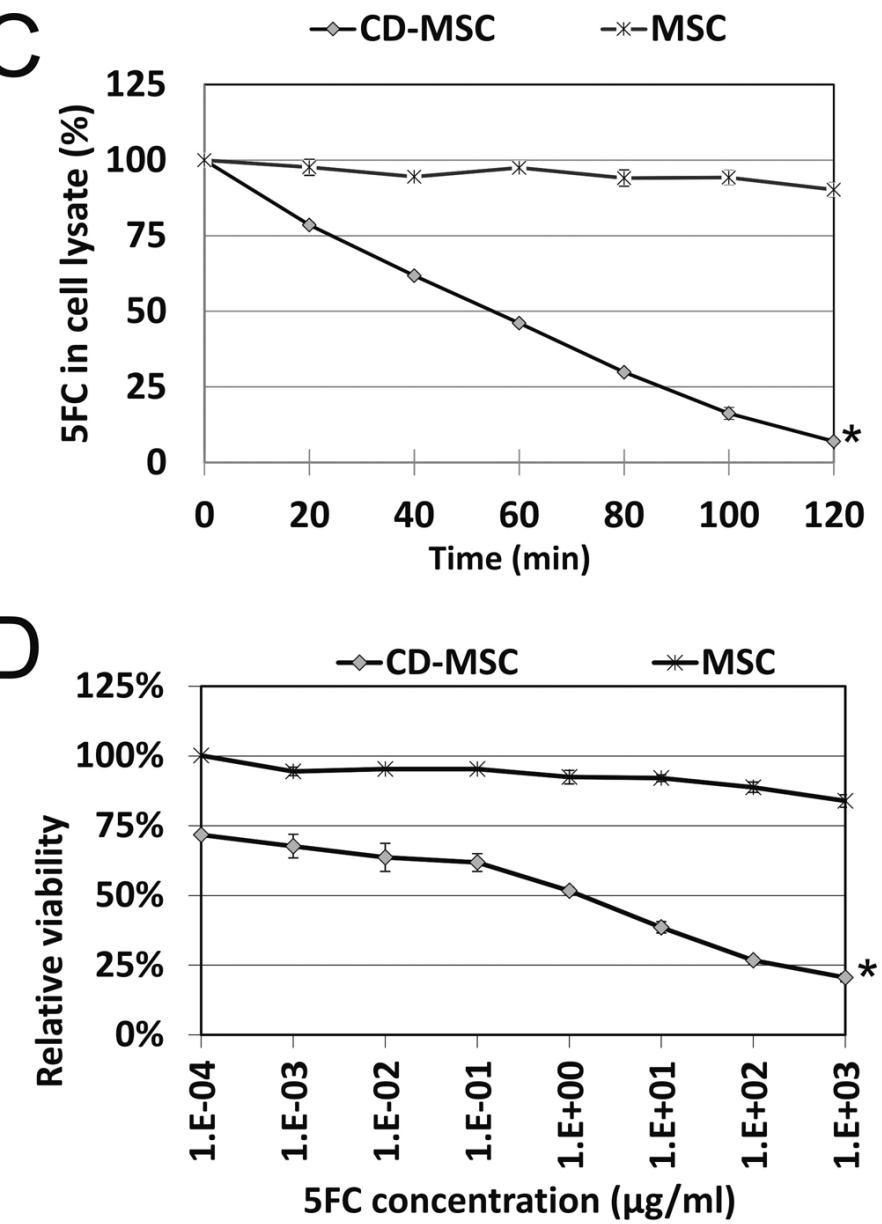

Figure 1. CD-MSC characterization. A) Genomic DNA was isolated from MSC and CD-MSC and subjected to the PCR with primers specific for the transgene. The gene sequence of CD was detected in the CD-MSC and not in the bulk MSC as expected. B) Total RNA from MSC and CD-MSC was isolated, DNase treated, reverse transcribed and subjected to the PCR amplification. The outcome shows the detection of transgene expression in CDMSC only. The expression of housekeeping gene GAPDH was used as a template integrity control, 1 or $2 \mu \mathrm{g}$ of the cDNA was used for reaction (lanes 1 and 3 or 2 and 4, respectively). C) Cell lysate from MSC and CD-MSC was prepared and mixed with 5FC solution. The aliquots from the reaction incubated at $37^{\circ} \mathrm{C}$ were quenched with $\mathrm{HCl}$ and the $5 \mathrm{FC}$ content was measured spectrophotometrically. CD-MSC lysate contained enzymatic activity converting $5 \mathrm{FC}$ as determined by gradual decrease of 5FC in the reaction mixture. D) MSC or CD-MSC (2,000/well) were plated in 96-well plates, let to adhere overnight and medium was replaced by the medium containing given $5 \mathrm{FC}$ concentration. MTS assay 5 days later detected significant decrease in the CD-MSC viability in comparison to the MSC, ${ }^{\star} \mathrm{p}<0.05$. 
Melanoma cells A375 layered on the agarose cushion were able to form tight compact nodule structures which could be maintained in the standard culture medium for several days (Fig. 2A). In order to compare the drug sensitivity of the same cells under different culture conditions, we have treated both melanoma monolayer cultures and nodules with 5FU. We have observed substantially lower induction of DNA laddering as an indicator of late stage of apoptosis in the 5FU-treated nodules versus monolayer cultures (Fig. 2B). Moreover, when evaluated the extent of the Caspase-3/7 activation, again we have observed that in the nodule structures the induction of apoptosis was hindered and the nodule structures were refractory to the $5 \mathrm{FU}$ treatment in vitro (Fig. $2 \mathrm{C}$ ).

As a next step, we have attempted to produce multicellular nodules from the therapeutic CD-MSC cells admixed to the target melanoma cells A375 similarly to the direct cocultures as performed in standard monolayer conditions. Initial experiments have confirmed the capability of these cells to form tight nodule structures which could be propagated for several days. Therefore we examined the capability of the therapeutic CD-MSC cells to mediate 5FC conversion as previously described by using cell lysates from cocultures of both monolayers and nodules. We were able to show that there is a significant decrease in 5FC in cocultures containing $32 \%$ of CD-MSC admixed to melanoma cells as soon as 2 hours after reaction started (Fig. 3A). More importantly, there was no significant difference in the conversion between monolayer and nodule cocultures which led us to the conclusion that the efficiency of prodrug conversion was not altered by $3 \mathrm{D}$ culture method. Next, both monolayer and nodule cocultures

\section{A Monolayer culture}

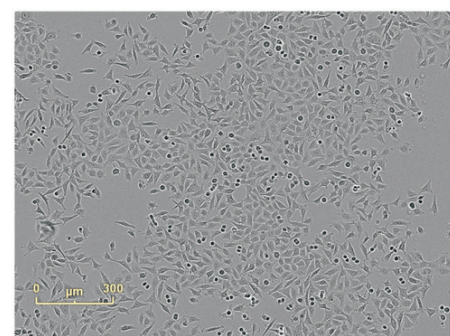

3D multicellular nodule

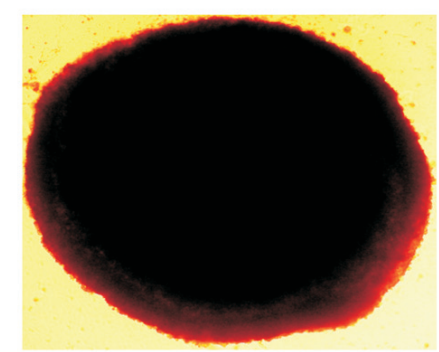

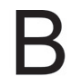

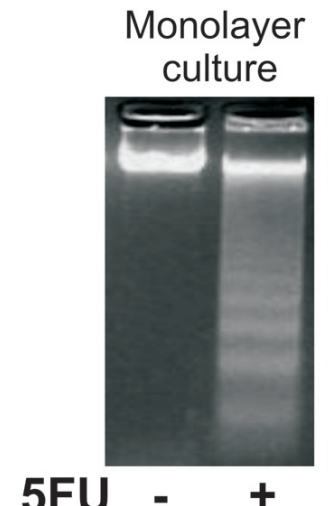

$3 \mathrm{D}$ nodule

C $\prec$ Monolayer $\quad \rightarrow$ 3D nodule
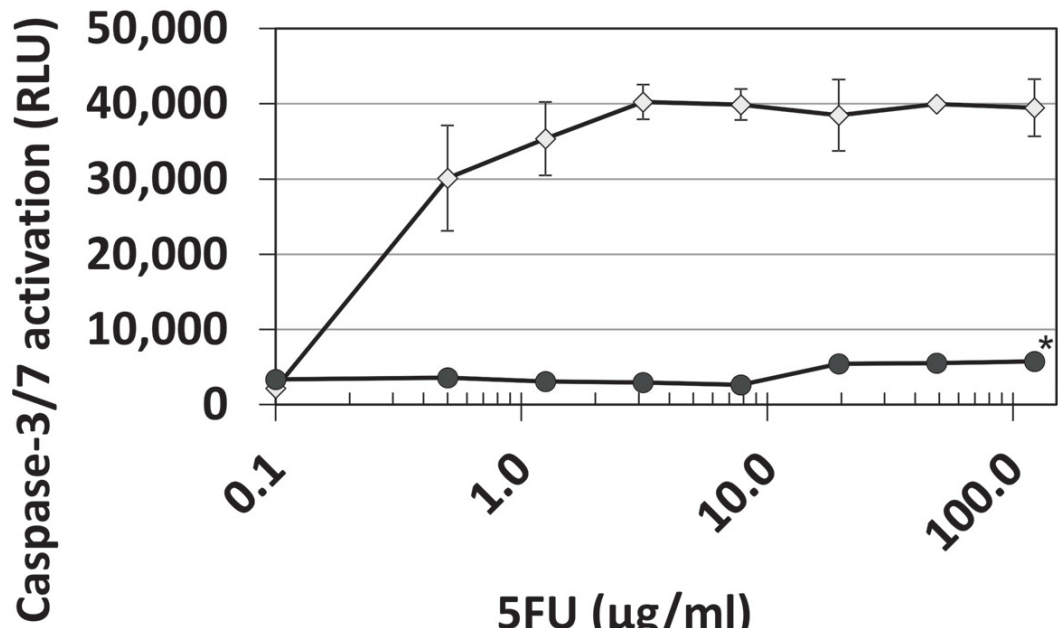

$5 \mathrm{FU}(\mu \mathrm{g} / \mathrm{ml})$

Figure 2. Chemoresistance of multicellular nodules. A) Phase contrast images of melanoma A375 cells demonstrating cellular morphology in monolayer cultures and a representative image of tight multicellular nodule structure 3 days after culture initiation. B) A375 cell were plated in monolayer or nodule cultures, $5 \mathrm{FU}$ treatment with $5 \mu \mathrm{g} / \mathrm{ml}$ 5FU in culture media was started next day. The cells were collected after 3 days, total DNA was isolated and $5 \mu \mathrm{g}$ was loaded on a $2 \%$ gel to visualize DNA fragmentation. The $5 \mathrm{FU}$ did not induce laddering in nodules, although the same treatment produced apoptotic DNA fragments in monolayer cultures. C) Monolayer and nodules were treated with given 5 FU concentration for 24 hours and the extent of Caspase-3/7 activity was determined by the luminescent assay. Nodule cultures were refractory to the cytotoxic action of $5 \mathrm{FU}$ in contrast to the monolayer cultures. 
were treated with or without prodrug 5FC, and the cellular viability was determined by the luminescence-based evaluation of ATP content three days later. Our data have shown significant decrease in the viability being proportional to the number of CD-MSC admixed to the melanoma cells (Fig. 3B). However, the outcome suggested that the cytotoxic effect is significantly lower in the nodule cocultures in comparison to the monolayer cultures. The same effect was observed in an independent assay, when fluorescently labelled target melanoma EGFP-A375 cells were subjected to the treatment as above, and the outcome was evaluated by measurement of green fluorescence directly corresponding to the number of remaining melanoma EGFP-positive cells in cocultures. Antitumor effect was much lower in nodule cultures as the relative fluorescence was decreased by only $21.0 \%$ in contrast to the monolayer cultures where the decrease achieved $84.5 \%$ when $20 \%$ of therapeutic CD-MSC cells was admixed to the cocultures (Fig. 3C). In order to confirm our findings we have used the advantage of recent technical developments which enabled us to monitor $3 \mathrm{D}$ cultures with kinetic method on a live-cell imaging platform IncuCyte $\mathrm{ZOOM}^{\mathrm{m}}$ with fluorescence optic [18]. We have plated the EGFP-A375 into ultra-low attachment $\mathrm{U}$ bottom 96 -well plates and we were able to observe the sphere formation and the growth as expected (data not shown). According to the kinetic observation based on the time-lapse images of individual spheroids we confirmed that the cocultures of melanoma with therapeutic CD-MSC cells produced growing spheroids under these conditions (Fig. 4A). Importantly, when the cocultures were supplemented with the prodrug $5 \mathrm{FC}$, significant gradual decrease in the spheroid volume was observed in the cocultures containing more than $5 \%$ of the CD-MSC (Fig. 4B). Spheroid shrinkage is an important translational paradigm for drug treatments and a spheroid volume measurement based on fluorescence intensity provides a surrogate indicator of cell health following pharmacological intervention. We have extended the analysis further and applied adopted modified protocol for the viability measurements based on the luminescent evaluation of ATP content in spheroid cultures at the experiment endpoint. Our data have shown
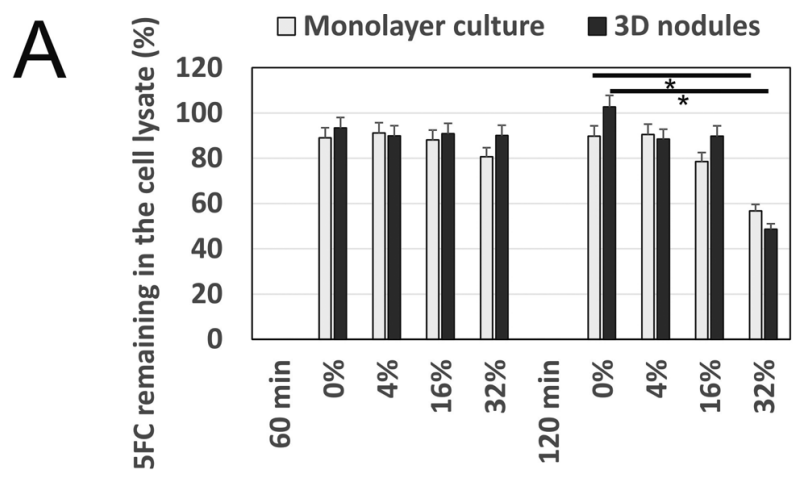

Proportion of CD-MSC cells in cocultures
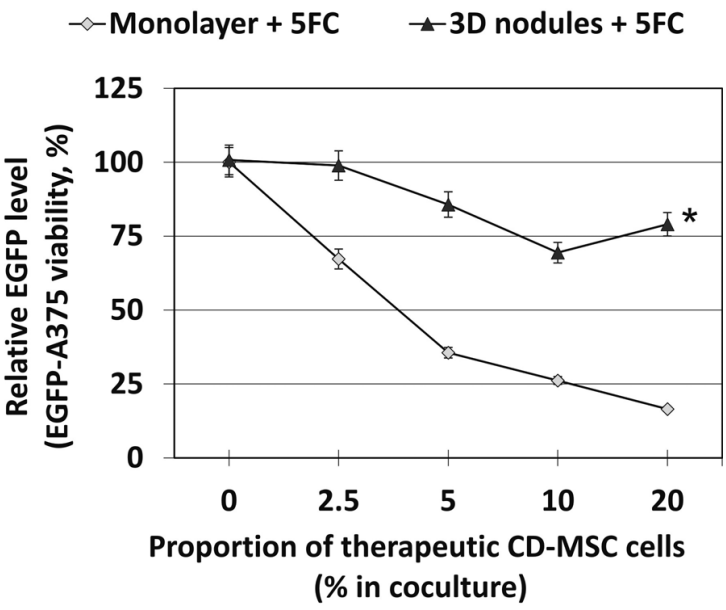

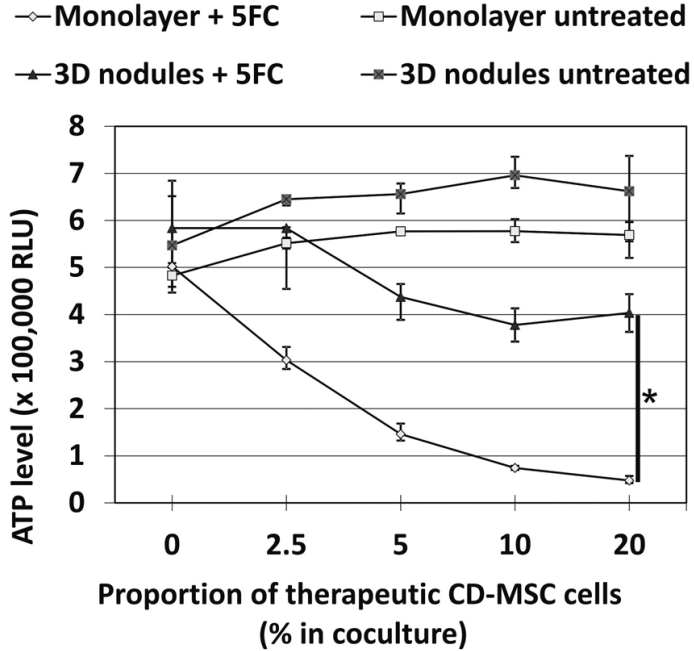

Figure 3. Bystander cytotoxicity in 3D nodules. A) Monolayer and nodule cultures were initiated from mixed therapeutic and tumor cells at given proportion. Cells were collected 3 days later, lysed and supplemented with 5FC. 5FC concentration in the reaction was determined spectrophotometrically. There was a significant decrease in the 5FC concentration after 2 hours of incubation in cocultures containing $32 \%$ of the CD-MSC $\left({ }^{*} \mathrm{p}<0.05\right)$ in comparison to melanoma cells only. No significant difference was detected between $5 \mathrm{FC}$ conversion in monolayer versus nodule coculture. B) Cultures were initiated as above and $24 \mathrm{hrs}$ later supplemented with $50 \mu \mathrm{g} / \mathrm{ml} 5 \mathrm{FC}$ containing medium where indicated. Relative ATP level was determined by luminescent measurement (RLU - relative luminescent units). Viability was significantly lower in the monolayer cultures in contrast to 5FC treated nodules $\left({ }^{*} p<0.05\right)$. C) Cultures were initiated with EGFP-A375 cells as above and $24 \mathrm{hrs}$ later supplemented with $50 \mu \mathrm{g} / \mathrm{ml}$ 5FC containing medium where indicated. Relative green fluorescence was determined by fluorimetric measurement. Data were calculated by dividing experimental value for $5 \mathrm{FC}$ treated with the value from untreated respective well with the same proportion of therapeutic to target cells and multiplied by 100 to get per cent. Relative fluorescence was significantly lower in 5FC-treated monolayer cultures in contrast to the nodules $\left({ }^{*} \mathbf{p}<0.05\right)$. 
that under these non-adherent 3D culture conditions more than $5 \%$ of the therapeutic cells were required in the coculture to achieve at least $50 \%$ decrease in relative viability of the cells (Fig. 4C). Nevertheless, we have clearly shown that $20 \%$ of the CD-MSC were able to achieve spheroid shrinkage within 48 hours of 5FC treatment, and 94\% decrease in viability after 5 days of the treatment thus corresponding to the previously observed data from the xenograft treatments in vivo $[6 ; 12]$. Based on these outcomes we concluded that
$3 \mathrm{D}$ culture methods can be used for better prediction of the efficiency of the cell-based enzyme/prodrug gene therapy on animal models. They represent a situation of the early stage non-vascularized tumors and better reflect the extent of the pharmacologic intervention in vivo. Our data suggested that the chemosensitivity in 3D nodules is lower in comparison to the monolayer cultures; and, moreover, the response to the given therapeutic regimen is also significantly lower in the $3 \mathrm{D}$ culture conditions.

A
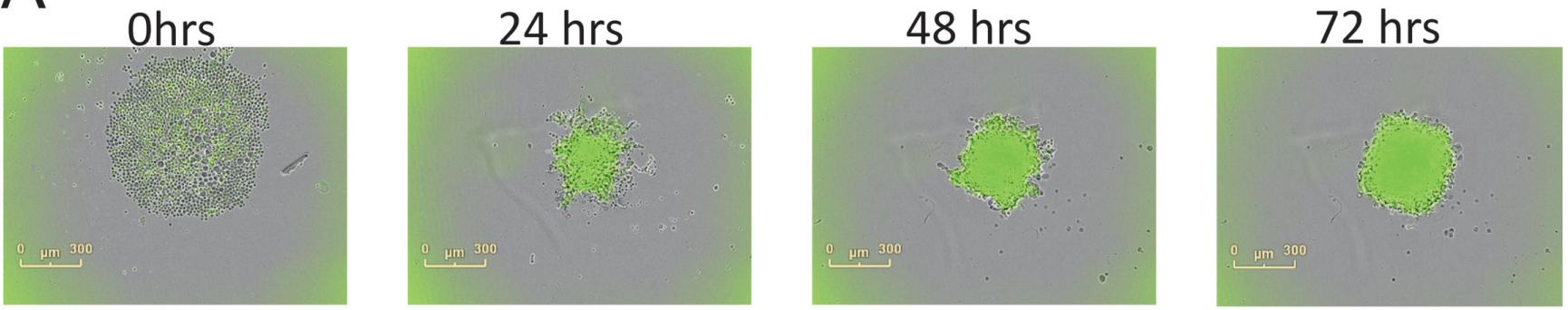

\section{D multicellular nodule in ultra-low attachment culture plates}
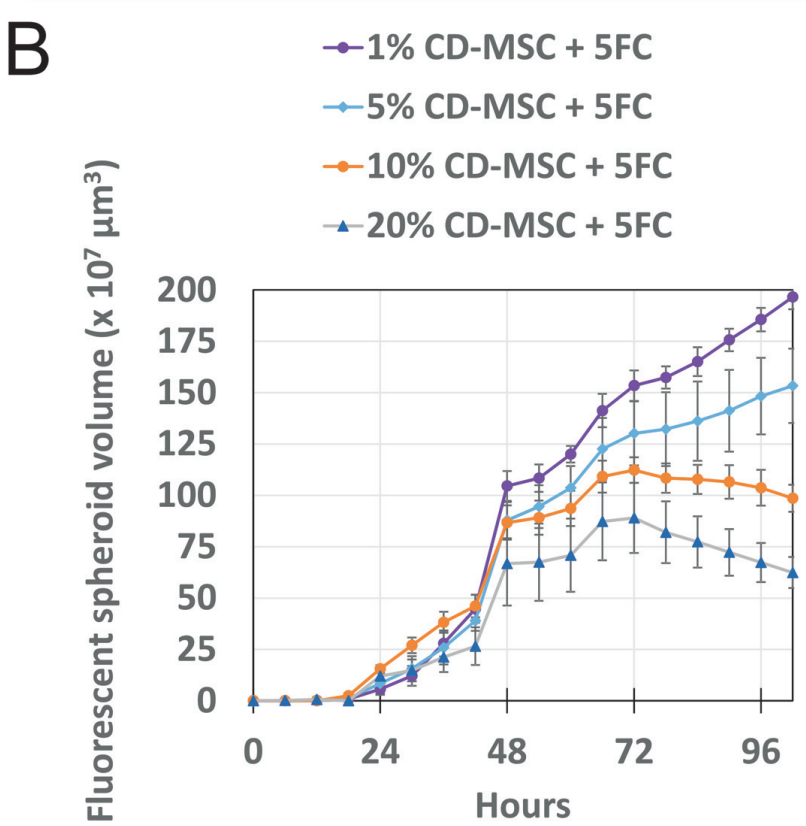

C

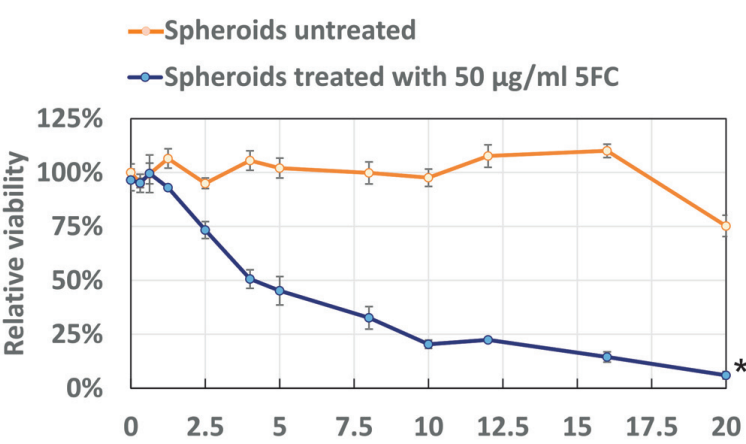

Proportion of therapeutic CD-MSC cells in cocultures (\%)

Figure 4. Kinetic measurements of bystander cytotoxicity in 3D spheroids. A) EGFP-A375 cells (4,000 per well) were mixed with the CD-MSC (400 per well) and seeded in ultra-low attachment U-bottom 96-well plates. Spheroid development was monitored by serial time-lapse images taken every 6 hour in the IncuCyte ZOOM ${ }^{\mathrm{m}}$ FLR. Representative images merged from phase-contrast and green fluorescent channels are shown to demonstrate spheroid formation 24 hours after plating and its gradual growth thereafter. B) Cells at different ratios were seeded for kinetic monitoring as above. $5 \mathrm{FC}$ ( 50 $\mu$ / $\mathrm{ml}$ ) was supplemented into the media 48 hrs later. Spheroid volume was calculated as described in CellPLayer ${ }^{\text {ta }}$ 96-well kinetic $3 \mathrm{D}$ spheroid protocol (Essen Bioscience). Spheroid growth in cocultures without 5FC was very similar to the curve for $1 \%$ of the CD-MSC +5 FC (violet color) and therefore omitted from the graph for clarity. The data show gradual decrease in the spheroid volume with a linear correlation to the proportion of CD-MSC in the spheroid cocultures. C) The kinetic experiment from the $4 \mathrm{~B}$ was ended 5 days after the 5FC treatment initiation. One hundred $\mu \mathrm{l}$ of media was discarded, the wells were supplemented with the luminescent reagent and after 10 min of shaking transferred to the white-walled plates. Luminescence directly proportional to the number of the viable cells has unravelled significant bystander cytotoxicity exerted by the CD-MSC in the presence of the $5 F C$ in $3 \mathrm{D}$ culture conditions, $\left({ }^{*} \mathrm{p}<0.05\right)$. 


\section{Discussion}

Human mesenchymal stromal cells (MSC) were suggested to represent suitable cellular delivery vehicles due to their preferential engraftment to the sites of tumor growth. Many researchers including our group have already confirmed that the adipose tissue-derived human MSC are advantageous in terms of their availability and expandability with no major biological hindrance over the MSC derived from other tissues, e.g. bone marrow. We have already reported the antitumor efficiency of the systemically administered engineered MSC in preclinical models of colon carcinoma and melanoma $[5 ; 6]$. Our present efforts are directed towards the evaluation of the efficiency on experimental metastatic models. The preliminary data have suggested the benefit of the CD-MSC/5FC for the survival and control of metastatic spread (manuscript under revision, M.M., and unpublished data, L.K.). However, our data also suggested the necessity of development of more advanced models for in vitro testing and a prediction of the therapeutic response. Several reports indicated previously that there were suitable approaches to the $3 \mathrm{D}$ cultures of engineered therapeutic and non-modified tumor cells. Initial studies on the MSC viability in 3D culture conditions in spheroids were performed with or without cartilage or scaffolds as the MSC were intended for the use in bone regeneration therapy. It has been shown that the MSC could be propagated as spheroids with no major impact on their viability, only glucose deprivation might substantially impact on the MSC viability in the 3D conditions [19; 20]. Our intention towards examination of $\mathrm{CD}-\mathrm{MSC} / 5 \mathrm{FC}$ regimen in $3 \mathrm{D}$ culture models was further encouraged by the work of Benouchan et al., who employed a three-dimensional multicellular nodule model in the genedirected enzyme/prodrug therapy (GDEPT) approach [15]. They demonstrated the bystander cell killing mediated by endothelial cells transfected with a suicide gene nitroreductase (NTR) on unmodified tumor cells by using a 3D multicellular nodule model. More recently, Hunt et al. presented coated spheroids as the 3D spatially distinct model to represent tissue-like cell density [21]. However, they observed absence of cytotoxic response in the $3 \mathrm{D}$ conditions in contrast to the monolayer cultures with the enzyme/prodrug NTR/CB1954 combination expressed in a human bladder carcinoma cells T24. These outcomes do not correspond to the previously published study showing that the bystander effect measured in three-dimensional multilayer cocultures of NTR+ and NTRcells was much more pronounced in 3D tumor cell cocultures [22]. Whether the opposite effect is due to the inherent properties of given model cell line or the methodological cues cannot be discriminated at this moment. Based on the data mining we have chosen to start with the protocol of multicellular nodules culture in the absence of any other component such as matrigel, basement membrane component etc. Our idea was to model the situation in the coinjected xenotransplants shortly after administration, as we have previously performed. We always injected the cells in the absence of any extracellular matrix in believe that it was the best recapitulation of the situation in our immunocompromised animals in vivo.

The report from the mixed 3D cultures with breast cancer cells and the MSC has proven the feasibility of the nodule growth in mixed setup [23]. Detailed analysis suggested that the MSCs rendered the breast cancer cells more susceptible to the kinase inhibitors in the 3D culture conditions. Previous report from Harma et al. showed that specific small molecule inhibitors targeted against PI3-kinase blocked invasive cell growth more effectively in $3 \mathrm{D}$ than in $2 \mathrm{D}$ monolayer culture of prostate cancer cells [24]. Aljitawi et al. compared the chemosensitivity of leukemia cells in a novel 3D stromal-based model and showed on contrary increased chemoresistance of the leukemic cells cocultured with stromal cells in non-adherent conditions [25]. Further correlations and investigation of different non-adherent models of cell proliferation will be useful for predictive testing of anti-cancer and anti-metastatic compounds. Nevertheless, the heterogeneity of the cellular responses depending on the models used in vitro and culture conditions is emerging. Melanoma cells A375 alone or mixed with the CD-MSC formed tight multicellular nodules both on the agarose cushion and in the ultra-low attachment conditions preventing cell adhesion and spread. Thus we could perform the experiments in both conditions and exploit the benefit from both of them. By two independent methods we have seen that the multicellular nodules remained refractory to $5 \mathrm{FU}$ in vitro. This is in a sharp contrast to the sensitivity of A375 melanoma cells from the monolayer cultures to $5 \mathrm{FU}$ [12]. It is well established that melanoma cells are chemoresistant and do not respond to the chemotherapy in clinical situation in general, including $5 \mathrm{FU}$-based regimens. The similar outcome was observed in our animal model, where mice bearing A375 xentransplants were injected with 5FU. No significant inhibition of the tumor growth could be achieved, thus we conclude that the 3D multicellular nodules closely recapitulate the situation in vivo. Therefore we believe that the $3 \mathrm{D}$ multicellular model is suitable to predict the therapeutic efficiency.

The outcomes of the coculture experiments, when the therapeutic CD-MSC were mixed with the target tumor cells further supported this notion. In each of the setups the monolayer cultures were significantly more sensitive to the bystander cytotoxicity exerted by the CD-MSC in the presence of prodrug 5FC. Importantly, the measurement of the 5FC conversion did not indicate compromised enzymatic activity in the CD-MSC incorporated in the 3D nodules (Fig. $3 \mathrm{~A})$. When compared to the monolayer cultures, previous data have shown that the $5 \%$ of the CD-MSC in the coculture decreased A375 cell viability by $90 \%$ within 5 days of the $5 \mathrm{FC}$ treatment. The viability measurement in the $3 \mathrm{D}$ non-adherent conditions revealed a decrease only by $50 \%$ (Fig. 4C). In order to diminish the viability to less than $90 \%$, there had to be the $20 \%$ of the CD-MSC present at the culture initiation. This outcome corresponds to the data already published, when $20 \%$ of CD-MSC/5FC were able to significantly reduce the tumor growth and achieve a complete tumor regression in the $83.3 \%$ 
of the animals in a long-term experiment [12]. Although the $10 \%$ of the therapeutic cells significantly reduced a spheroid volume and achieved the $75 \%$ inhibition of the viability, this was sufficient for the tumor inhibition but not for the complete tumor eradication in animals.

In summary, based on these data we have shown for the first time a suitable 3D model for the MSC-directed enzyme/ prodrug therapy approaches. The treatment efficiencies could be directly correlated to the outcomes achieved in vivo. We have shown here that the monolayer cultures overestimate the treatment sensitivity. We propose to examine the potential application of the cell-directed enzyme/prodrug therapy on specific tumor cells in the $3 \mathrm{D}$ cultures first, based on the prerequisite that the cells can grow as tight nodules under these conditions. Alternatively, another components of the tumor microenvironment such as matrigel or ECM can be tested for their contribution to the treatment efficiency. Based on our data, the best outcomes with the CD-MSC/5FC regimen might be expected in the tumors which incorporate substantial part of the tumor stroma from the adipose tissuederived precursors (such as ovarian cancer and breast cancer models) - ideally close to $20 \%$ [26; 27]. It still remains to be determined whether the given regimen is capable of controlling or eradicating metastatic spread, where the systemic administration of the MSC might enable to achieve high local tissue engraftment specifically as the first-pass effect in particular organ.

Acknowledgements: We acknowledge the excellent technical help and assistance from M. Dubrovcakova and V. Frivalska. This work was supported by the Slovak Research and Development Agency under the contract No. APVV-0230-11, APVV-0052-12, VEGA grants $2 / 0087 / 15,2 / 0171 / 13$ and $2 / 0130 / 13$. The experiments on the IncuCyte $\mathrm{ZOOM}^{\mathrm{rw}}$ were enabled with the kind help and the financial support from the Cancer Research Foundation and RFL2011.

\section{References}

[1] KEATING A Mesenchymal stromal cells: new directions. Cell stem cell 2012; 10: 709-716. http://dx.doi.org/10.1016/j. stem.2012.05.015

[2] STRIOGA M, VISWANATHAN S, DARINSKAS A, SLABY O, MICHALEK J Same or not the same? Comparison of adipose tissue-derived versus bone marrow-derived mesenchymal stem and stromal cells. Stem Cells Dev 2012; 21: 2724-2752. http://dx.doi.org/10.1089/scd.2011.0722

[3] HONG IS, LEE HY, KANG KS Mesenchymal stem cells and cancer: Friends or enemies? Mutation research. Fundamental and molecular mechanisms of mutagenesis 2014. http://dx.doi. org/10.1016/j.mrfmmm.2014.01.006

[4] DURINIKOVA E, KUCEROVA L, MATUSKOVA M Mesenchymal stromal cells retrovirally transduced with prodrug-converting genes are suitable vehicles for cancer gene therapy. Acta virologica 2014; 58: 1-13. http://dx.doi. org/10.4149/av $2014 \quad 01 \quad 3$
[5] KUCEROVA L, ALTANEROVA V, MATUSKOVA M, TYCIAKOVA S, ALTANER C Adipose tissue-derived human mesenchymal stem cells mediated prodrug cancer gene therapy. Cancer Res 2007; 67: 6304-6313. http://dx.doi. org/10.1158/0008-5472.CAN-06-4024

[6] KUCEROVA L, MATUSKOVA M, PASTORAKOVA A, TYCIAKOVA S, JAKUBIKOVA J, et al. Cytosine deaminase expressing human mesenchymal stem cells mediated tumour regression in melanoma bearing mice. J Gene Med 2008; 10: 1071-1082. http://dx.doi.org/10.1002/jgm.1239

[7] KIDD S, SPAETH E, DEMBINSKI JL, DIETRICH M, WATSONK, et al. Direct evidence of mesenchymal stem cell tropism for tumor and wounding microenvironments using in vivo bioluminescent imaging. Stem Cells 2009; 27: 2614-2623. http://dx.doi.org/10.1002/stem.187

[8] KUCEROVA L, SKOLEKOVA S, MATUSKOVA M, BOHAC $\mathrm{M}$, KOZOVSKA Z Altered features and increased chemosensitivity of human breast cancer cells mediated by adipose tissue-derived mesenchymal stromal cells. BMC Cancer 2013; 13: 535. http://dx.doi.org/10.1186/1471-2407-13-535

[9] CIHOVA M, ALTANEROVA V, ALTANER C Stem cell based cancer gene therapy. Mol Pharm 2011; 8: 1480-1487. http:// dx.doi.org/10.1021/mp200151a

[10] ALTANER C Prodrug cancer gene therapy. Cancer Lett 2008; 270: 191-201. http://dx.doi.org/10.1016/j.canlet.2008.04.023

[11] MATUSKOVA M, BARANOVICOVA L, KOZOVSKA Z, DURINIKOVA E, PASTORAKOVA A, et al. Intrinsic properties of tumour cells have a key impact on the bystander effect mediated by genetically engineered mesenchymal stromal cells. J Gene Med 2012; 14: 776-787. http://dx.doi. org/10.1002/jgm.2684

[12] KUCEROVA L, SKOLEKOVA S, DEMKOVA L, BOHOVIC $\mathrm{R}$, MATUSKOVAM Long-term efficiency of mesenchymal stromal cell-mediated CD-MSC/5FC therapy in human melanoma xenograft model. Gene Ther 2014; 21: 874-887. http://dx.doi.org/10.1038/gt.2014.66

[13] KUCEROVA L, MATUSKOVA M, HLUBinOVA K, ALTANEROVA V, ALTANER C Tumor cell behaviour modulation by mesenchymal stromal cells. Mol Cancer 2010; 9: 129. http://dx.doi.org/10.1186/1476-4598-9-129

[14] KUCEROVA L, ZMAJKOVIC J, TORO L, SKOLEKOVA S, DEMKOVA L, MATUSKOVA M Tumor-driven Molecular Changes in Human Mesenchymal Stromal Cells. Cancer Microenviron 2014. http://dx.doi.org/10.1007/s12307-014-0151-9

[15] BENOUCHAN M, DO NASCIMENTO F, SEBBAH-LOURIKI M, SALZMANN JL, CREPIN M, et al. Bystander cell killing spreading from endothelial to tumor cells in a threedimensional multicellular nodule model after Escherichia coli nitroreductase gene delivery. Biochem Biophys Res Commun 2003; 311: 822-828. http://dx.doi.org/10.1016/j. bbrc.2003.10.068

[16] HLAVATY J, HLUBINOVA K, ALTANER C Construction and testing of gene therapy retroviral vector expressing bacterial cytosine deaminase gene. Neoplasma 1999; 46: 267-276.

[17] ZHANG Y, WU LJ, TASHIRO S, ONODERA S, IKEJIMA $\mathrm{T}$ Evodiamine induces tumor cell death through different 
pathways: apoptosis and necrosis. Acta Pharmacologica Sinica 2004; 25: 83-89.

[18] VINCI M, GOWAN S, BOXALL F, PATTERSON L, ZIMMERMANN M, et al. Advances in establishment and analysis of three-dimensional tumor spheroid-based functional assays for target validation and drug evaluation. Bmc Biology 2012; 10. http://dx.doi.org/10.1186/1741-7007-10-29

[19] FARRELL MJ, SHIN JI, SMITH LJ, MAUCK RL Functional consequences of glucose and oxygen deprivation on engineered mesenchymal stem cell-based cartilage constructs. Osteoarthritis Cartilage 2014.

[20] FRITH JE, THOMSON B, GENEVER PG Dynamic threedimensional culture methods enhance mesenchymal stem cell properties and increase therapeutic potential. Tissue Eng Part C Methods 2010; 16: 735-749. http://dx.doi.org/10.1089/ten. tec.2009.0432

[21] HUNT MA, LI D, HAY MP, CURRIE MJ, ROBINSON BA, et al. Characterisation of enzyme prodrug gene therapy combinations in coated spheroids and vascular networks in vitro. J Gene Med 2012; 14: 62-74. http://dx.doi.org/10.1002/igm.1635

[22] WILSON WR, PULLEN SM, HOGG A, HELSBY NA, HICKS $\mathrm{KO}$, et al. Quantitation of bystander effects in nitroreductase suicide gene therapy using three-dimensional cell cultures. Cancer Research 2002; 62: 1425-1432.
[23] DITTMER A, FUCHS A, OERLECKE I, LEYH B, KAISER $S$, et al. Mesenchymal stem cells and carcinoma-associated fibroblasts sensitize breast cancer cells in $3 \mathrm{D}$ cultures to kinase inhibitors. International Journal of Oncology 2011; 39: 689-696. http://dx.doi.org/10.3892/ijo.2011.1073

[24] HARMA V, VIRTANEN J, MAKELA R, HAPPONEN A, MPINDI JP, et al. A Comprehensive Panel of Three-Dimensional Models for Studies of Prostate Cancer Growth, Invasion and Drug Responses. PLoS One 2010; 5.

[25] ALJITAWI OS, LI DD, XIAO YH, ZHANG D, RAMACHANDRAN K, et al. A novel three-dimensional stromal-based model for in vitro chemotherapy sensitivity testing of leukemia cells. Leukemia Lymphoma 2014; 55: 378-391. http://dx.doi. org/10.3109/10428194.2013.793323

[26] KIDD S, SPAETH E, WATSON K, BURKS J, LU H, et al. Origins of the tumor microenvironment: quantitative assessment of adipose-derived and bone marrow-derived stroma. PLoS One 2012; 7: e30563. http://dx.doi.org/10.1371/journal. pone. 0030563

[27] SPAETH EL, DEMBINSKI JL, SASSER AK, WATSON K, KLOPP A, et al. Mesenchymal stem cell transition to tumorassociated fibroblasts contributes to fibrovascular network expansion and tumor progression. PLoS One 2009; 4: e4992. http://dx.doi.org/10.1371/journal.pone.0004992 